\title{
Time in Cyber-Physical Systems Specifications, Modeling and Measurements
}

\author{
Miroslav Sveda \\ Faculty of Information Technology \\ Brno University of Technology \\ Brno, Czech Republic \\ e-mail: sveda@ fit.vutbr.cz
}

Received: July 20, 2019. Revised: October 5, 2021. Accepted: October 27, 2021. Published: November 23, 2021.

\begin{abstract}
This paper addresses the role, interpretation and the deployment of the notion "time" in distributed cyber-physical systems. It discusses various possibilities how to approach such modeling and selects the fitting one, which enables to utilize the related specification language ASL in the domain applications.
\end{abstract}

Keywords-cyber-physical system; time; temporal partial order; operational semantics; measurement.

\section{INTRODUCTION}

The integration of physical systems and processes with networked computing has led to the emergence of a new generation of engineered systems: Cyber-Physical Systems (CPS) [9]. Such systems use computations and communication deeply embedded in and interacting with physical processes to add new capabilities to physical systems. These cyber-physical systems range from small embedded applications, such as pace makers to large-scale huge systems, e.g. the international power-grid. Because computer-augmented devices are everywhere, they are a huge source of economic leverage. Embedded computers allow designers to add capabilities to physical systems that they could not feasibly add in any other way. By merging computing and communication with physical processes and mediating the way how to interact with the physical world [14], cyber-physical systems bring many benefits: they make systems safer and more efficient; they reduce the cost of building and operating these systems; and they allow individual machines to work together to form complex systems that provide new capabilities. By merging computing and communication with physical processes and mediating the way we interact with the physical world, cyber-physical systems bring many benefits: they make systems safer and more efficient, they reduce the cost of building and operating these systems, and they allow individual machines to work together to form complex systems that provide new capabilities.

This paper considers the orchestration of computing with physical processes. It argues that to realize its full potential, the core abstractions of computing need to be rethought to incorporate essential properties of the physical systems, most particularly the passage of time [13], [4].

The kernel of the paper consists of an explanation of the notion "time" in sections II. and III., and of presenting the Asynchronous Specification Language (ASL) including its operational semantics for temporal partial order in sections IV. and V. Next section discusses two case studies, the first one demonstrates using ASL for behavioral specification of lift cabin position measurement, and the second one time measurement with clock synchronization in a distributed system based on Internet.

\section{TIME}

Norbert Wiener in the Chapter 1, Newtonian and Bergsonian Time, of his book [22] distinguishes between reversible, Newtonian time of classical mechanics and irreversible time of cybernetics with definite past-future order fitting also such disciplines as meteorology, thermodynamics, statistical mechanics, and biology. Physicists perfect this notion into thermodynamic, psychological, and cosmological time arrows that point in the same direction [5]. Contemporary Cybernetics deals -- in frame of its branches such as Artificial Intelligence, Systems Theory, or Software Engineering -- with various concepts and refinements of directed time. This paper reviews those concepts and brings examples of their applications.

Basic meanings of the term "time" can be introduced in the following complementary couples: physical/logical, absolute/relative, global/local. To be more precise, we consider an event domain, E, and a time domain, T, such that instead of viewing the precedence relation "to causally affect" on events we use members of a time domain to mark the members of the event domain to introduce a temporal order [12]. Especially, the physical time means that passing of time is the primary cause for anything to happen; actually, it denotes counting cycles of a physical, strictly periodic process [1]. The logical time means that time passes only because something happens -- it respects order of events only [8]. The absolute time means that a reference is established in relation to a unique event for a given system; evidently, it relates to some origin of date/time, see e.g. [10]. The relative time means that a reference is established in relation to an 
arbitrary selected event in the given system; clearly, it relates to time intervals. The global time means that the time is considered to be valid for the whole (distributed) system while the local time means that the time is valid for a part of the (distributed) system.

Various disciplines utilize several notions of time. To be more complete, we should specify time models by more attributes than precedence relation. Models of time can be classed, see e.g. [8], according to individuals (points, intervals), order (partial order, branching towards future, linear), boundedness (unbounded, beginning, ending), local structure (discrete, dense, continuous) and global structure (connectedness, homogeneity). Whereas synchronous models of computation regard all concurrent activities happen in a lock-step, asynchronous models are not restricted in this sense. They can be treated as interleaving models of computation, which sequentialize simultaneous actions non-deterministically, or as true concurrency models of computation, which impose only a partial ordering between actions. By the way, various models were designed aiming to describe also some relativistic phenomena, see e.g. [3].

An implicit time domain of a system process respects internal events (changes in the state) of that process. An explicit time domain, on the other hand, consists of events that are not produced in the process, but which bear an observable temporal relation of the local process [6]. Both types of timing can be considered as either internal to the local process or external to the remote processes (e.g. environmental processes). Evidently, implicit timing suffices only for a synchronous system timed by a common global clock or for a system driven by only one sequential process while real-time asynchronous distributed systems require explicit time domains. According to Holt [7], a model of real-time systems is natural if its internal time corresponds well with the external, physical time of the environment. However, different timing mechanisms rule various parallel environmental processes. In addition, distributed applications consider a distinctive, locally measured time for each node. A useful time model, therefore, must conform with external events as well as with internal timing, and it should provide unambiguous semantics for a specification and implementation of real-time distributed systems.

\section{SPACE AND Time Domains}

This subsection selects and narrows some ideas from [8], and [24] focusing on local time. The treatment of event-time relationships resembles to the approach presented in [15]; however, time domain is shifted from total order to partial order in this case. Hence, we consider an event domain, E, and a time domain, $T$, such that instead of viewing the precedence relation "to causally affect" on events we use members of a time domain to mark the members of the event domain to introduce a temporal order.

For each of the domains $\mathrm{E}$ and $\mathrm{T}$ there are two possibilities how to choose domain elements: points and intervals. To preserve simplicity, we select points for both domains. Consequently, events can be interpreted as changes of system states and members of time domain as time instants. In this case, timing of events is mapping $\mathrm{E} \rightarrow \mathrm{T}$. Actions with non-zero duration can be described by their starting and ending points that require individual timings. Point structures of domains $\mathrm{E}$ and $\mathrm{T}$ simplify introduction of partial order in general. Local time concept requires to employ a partial order consistent with locality either of events or of timing. There are at least two natural possibilities how to introduce a timed partial order on events: (a) to define locality as an equivalence relation on events Loc $=(\mathrm{E}, \sim)$ and then, for each class of that equivalence to specify a separate linear time, i.e. to use multiple time lines (see similar conception in [2]); or (b) to connect locality explicitly with temporal partial order $(\operatorname{Loc} \times \mathrm{T}, \sim)$ (see e.g. [23]). From the application viewpoint, both possibilities correspond to the same relation: for case (a), temporal partial order is induced on $\mathrm{T}$ by manifold mapping; for case (b), partially ordered time generates a decomposition of event domain into classes so that events in each class are mutually comparable by linear temporal order. Nevertheless, we prefer the case (a).

Koymans [8] distinguishes three local temporal structures: discrete, dense, and continuous. The same can be applied to space or even space-time coordinates, see e.g. [3]. We prefer scalable discrete time structure and fix discrete finite space structure in form of finite set of locations.

An implicit time domain of a system process respects internal events (changes in the state) of that process. An explicit time domain, on the other hand, consists of events that are not produced in the process, but which bear an observable temporal relation of the local process [6]. Both types of timing can be considered as either internal to the local process or external to the remote processes (e.g. environmental processes). Evidently, implicit timing suffices only for a synchronous system timed by a common global clock or for a system driven by only one sequential process while real-time (asynchronous) distributed systems require explicit time domains. In accordance with Holt [7], a model of real-time systems is natural if its internal time corresponds well with the external, physical time of the environment. However, different timing mechanisms rule various parallel environmental processes. In addition, distributed applications consider a distinctive, locally measured time for each node. A useful time model, therefore, must conform with external events as well as with internal timing, and it should provide unambiguous semantics for a specification and implementation of real-time distributed systems.

\section{OPERATIONAL SEMANTICS FOR TEMPORAL PARTIAL ORDER}

The particular behavior of a of a non-Zeno, discrete realtime system can be described by an infinite sequence of pairs of states $s_{i}$ and corresponding times $t_{i}[2]$ :

$$
\mathrm{P}: \quad\left(\mathrm{s}_{0}, \mathrm{t}_{0}\right) \rightarrow\left(\mathrm{s}_{1}, \mathrm{t}_{1}\right) \rightarrow\left(\mathrm{s}_{2}, \mathrm{t}_{2}\right) \rightarrow \ldots
$$

Different models of time interpret the time component, $t$, of the system behavior, $\mathrm{P}=(\mathrm{s}, \mathrm{t})$, in different ways. While interval models of time associate each state with its duration 
over time, clock models stamp observations of the node state with time instants. To characterize asynchronous systems, whose node state changes can be arbitrarily close in time, analog-clock models record the exact time of every state. By contrast, digital-clock models measure the time of a state only with finite precision, approximating a dense time domain by a sequence of discrete values--the time between successive states may remain the same or may increase by an arbitrary amount. For a distributed system, its state space can be decomposed into the state spaces of its nodes $1,2, \ldots, n$ :

$$
\begin{aligned}
& { }^{1} \mathrm{P}:\left({ }^{1} \mathrm{~s}_{0},{ }^{1} \mathrm{t}_{0}\right) \rightarrow\left({ }^{1} \mathrm{~s}_{1},{ }^{1} \mathrm{t}_{1}\right) \rightarrow\left({ }^{1} \mathrm{~s}_{2},{ }^{1} \mathrm{t}_{2}\right) \rightarrow \ldots \\
& { }^{2} \mathrm{P}:\left({ }^{2} \mathrm{~s}_{0},{ }^{2} \mathrm{t}_{0}\right) \rightarrow\left({ }^{2} \mathrm{~s}_{1},{ }^{2} \mathrm{t}_{1}\right) \rightarrow\left({ }^{2} \mathrm{~s}_{2},{ }^{2} \mathrm{t}_{2}\right) \rightarrow \ldots \\
& { }^{\mathrm{j}} \mathrm{P}:\left({ }^{\mathrm{j}} \mathrm{s}_{0},{ }^{\mathrm{j}} \mathrm{t}_{0}\right) \rightarrow\left({ }^{\mathrm{j}} \mathrm{s}_{1},{ }^{\mathrm{j}} \mathrm{t}_{1}\right) \rightarrow\left({ }^{\mathrm{j}} \mathrm{s}_{2},{ }^{\mathrm{j}_{2}}\right) \rightarrow \ldots \\
& { }^{\mathrm{n}} \mathrm{P}:\left({ }^{\mathrm{n}} \mathrm{s}_{0},{ }^{\mathrm{n}} \mathrm{t}_{0}\right) \rightarrow\left({ }^{\mathrm{n}} \mathrm{s}_{1},{ }^{\mathrm{n}} \mathrm{t}_{1}\right) \rightarrow\left({ }^{\mathrm{n}} \mathrm{s}_{2},{ }^{\mathrm{n}} \mathrm{t}_{2}\right) \rightarrow \ldots
\end{aligned}
$$

In this case, additional attributes of time clarify the nature of the time component, ${ }^{j} t$, of the node $j$ 's behavior, ${ }^{\mathrm{j}} \mathrm{P}=\left({ }^{\mathrm{j}} \mathrm{s},{ }^{\mathrm{j}} \mathrm{t}\right)$ : real-time distributed architecture enrich models of time by considering the number of time lines. A single time line suffices for global clocks while multiple time lines support independent local clocks. Accordingly, the values ${ }^{j_{i}}$ and ${ }^{k_{i}}$ are either the $i$-th readings of global time, $t$, in nodes $j$ and $\mathrm{k}$ or the $\mathrm{i}$-th readings of local times ${ }^{\mathrm{j}} \mathrm{t}_{\text {and }} \mathrm{k}_{\mathrm{t}}$ in nodes $\mathrm{j}$ and $\mathrm{k}$. To respect the implementation viewpoint, distributed applications consider for each node a distinctive local time, i.e. the time of a local physical clock that suits to measuring a duration of local process actions.

Local time represents a concept of physical timing; still, its semantics can be derived from logical time and a physical generator of periodic events. In his pioneer work [11], Lamport defines logical time in a distributed system as a partial ordering of events in the system. Similarly for the purpose of this paper, time ordering of events in a system $\mathrm{S}$ is specified by a minimal partial-order relation $\rightarrow$ ("precedes") on events, which satisfies the following four conditions:

- if $\mathrm{A}$ and $\mathrm{B}$ are events in the same process and $\mathrm{A}$ is executed before $\mathrm{B}$, then $\mathrm{A} \rightarrow \mathrm{B}$ (the term "process" means sequential ordering of internal events);

- if $\mathrm{A}$ is the transmission of information by one process and $\mathrm{B}$ is the receipt of that information in $\mathrm{S}$, then $\mathrm{A} \rightarrow$ $\mathrm{B}$ (communication proceeds in non-zero time);

- if $\mathrm{A} \rightarrow \mathrm{B}$ and $\mathrm{B} \rightarrow \mathrm{C}$ in $\mathrm{S}$, then $\mathrm{A} \rightarrow \mathrm{C}$ (transitivity); and

- for any event $\mathrm{A}$ of $\mathrm{S}, \mathrm{A} \rightarrow \mathrm{A}$ does not hold $(\mathrm{A} \rightarrow \mathrm{A})$ (antireflexivity).

The totalization of the reflexive closure of the relation $\rightarrow$ ("precedes") is the relation $\Rightarrow$ ("precedes or is concurrent"):

- if $\mathrm{A} \nrightarrow \mathrm{B}$ in $\mathrm{S}$, then $\mathrm{B} \Rightarrow \mathrm{A}$.
An eventcount, E, is an object that counts the number of events of a specific type that have occurred during the execution of the system. Each such event occurrence invokes the operation ADVANCE(E): $\mathrm{E}:=\mathrm{E}+1$. The external operation $\operatorname{AWAIT}(E, \sigma)$ suspends the calling process until the value of the eventcount, E, is at least $\sigma$--the call $\operatorname{AWAIT}(\mathrm{E}, \sigma)$ usually resets the current value of $\mathrm{E}$, so the relative timing is possible. The external and internal operations interplay in the following way:

- if WE is the execution of the AWAIT operation in the form $\operatorname{AWAIT}(\mathrm{E}, \sigma)$, then there are at least $\sigma$ members of the set $\{\mathrm{AE} \mid \mathrm{AE}$ is the execution of $\mathrm{ADVANCE}(\mathrm{E})$ and $\mathrm{AE} \Rightarrow \mathrm{WE}\}$.

An eventcount can monitor the external events of a class that represents local physical timing of a distinctive part of the system environment. Also, periodic events implemented by an internal timer/counter circuit can advance an eventcount that tracks local-time clock events. So, the localtime model relates internal and accessible external clocking while internal local times in different nodes of a distributed system flow independently, without regular synchronization.

\section{ASYNCHRONOUS SPECIFICATION LANGUAGE}

The designed process-oriented procedural specification language includes primitives related to synchronization, timing, and communication. Hence, the specification of a system logical structure employs sequential processes, communicating asynchronously by message passing. A process represents the sequence of statements executed at a node of a distributed system. True concurrency with maximal parallelism is supposed: each process drives its own node; if a process is suspended, its node remains idle. Moreover, timing mechanisms of the processes are expressed with the help of local timers, using properly chosen time scales.

From the syntax viewpoint, the language for process specifications can be surveyed as an extended Pascal. The most important added primitives relate to process

process name(is:list_of_s_inputs; os:list_of_s_outputs; ic:list_of_m_inputs; oc:list_of_m_outputs):

... endprocess;

wait(_timeout); wait(event,_); wait(event,timeout,test); send(message,destination);

loop ... [... when $<$ cond $>$ action ... exit $]^{*}$... endloop;

specification, timing, communication, and control structure:

In this case, additional attributes of time clarify the nature of the time component, ${ }^{j}$, of the node $j$ 's behavior, ${ }^{\mathrm{j}} \mathrm{P}=\left({ }_{\mathrm{j}}^{\mathrm{j}} \mathrm{s},{ }_{\mathrm{t}}^{\mathrm{j}}\right)$ : real-time distributed architecture enrich models of time by considering the number of time lines. A single time line suffices for global clocks while multiple time lines support independent local clocks. Accordingly, the values ${ }^{j_{i}}$ and ${ }^{{ }^{k}} t_{i}$ are either the $i$-th readings of global time, $t$, in nodes $j$ and $k$ or the $i$-th readings of local times ${ }^{j}$ and ${ }^{k_{t}}$ in nodes $j$ and $\mathrm{k}$. To respect the implementation viewpoint, distributed 
applications consider for each node a distinctive local time, i.e. the time of a local physical clock that suits to measuring a duration of local process actions. In this case, additional attributes of time clarify the nature of the time component, ${ }_{t}$, of the node $\mathrm{j}^{\prime}$ s behavior, ${ }^{j} \mathrm{P}=\left({ }^{\mathrm{j}} \mathrm{s}\right.$, $\left.{ }^{\mathrm{j}} \mathrm{t}\right)$ : real-time distributed architecture enrich models of time by considering the number of time lines. A single time line suffices for global clocks while multiple time lines support independent local clocks. Accordingly, the values ${ }^{j} t_{i}$ and ${ }^{k} t_{i}$ are either the $i-t h$ readings of global time, $t$, in nodes $j$ and $k$ or the $i$-th readings of local times ${ }^{j_{t}}$ and $k_{t}$ in nodes $j$ and $k$. To respect the implementation viewpoint, distributed applications consider for each node a distinctive local time, i.e. the time of a local physical clock that suits to measuring a duration of local process actions.

Each of asynchronous processes can be equipped by its individually timed local clock, can receive messages through input buffer, and can send messages to other, directly or indirectly addressable processes. Process header contains in parentheses lists labelled by is, os, ic, and $o c$ that act as the interface with the process' environment. The language distinguishes between signal inputs or outputs, which denote un-buffered events carrying either value or signalling their occurrence, and message inputs or outputs as typed asynchronous channels between couples of processes. Those signals and messages declare the inter-process synchronization and communication, which operations are driven by the statements wait(event,_), wait(event,timeout,test), and send(message,destination).

The primitive wait(_timeout) suspends a process for the interval defined by the value timeout. Operational semantics can be obtained through the eventcount abstraction introduced above: in this case, an event is every tick of the local clock, so the related operation is AWAIT(local_ticks,timeout_value). For the primitive wait(event, ), which suspends a process until the specified event (external signal or message) appears, the model operation is AWAIT(event_type,1). The semantics of the combined statement wait(event,timeout,test) requires two eventcounts: the first anticipates the specified event and the second, with a lower priority, monitors the local clock. The reason of process activation can be checked through the value of the logical variable test: when the value is true, the event occurred within the interval timeout. In this case, additional attributes of time clarify the nature of the time component, ${ }^{j}$, of the node j's behavior, ${ }^{j} \mathrm{P}=\left({ }^{j} s,{ }^{j} t\right)$ : real-time distributed architecture enrich models of time by considering the number of time lines. A single time line suffices for global clocks while multiple time lines support independent local clocks. Accordingly, the values ${ }^{{ }^{j}} t_{i}$ and ${ }^{k_{t}}$ are either the $i$-th readings of global time, $t$, in nodes $j$ and $k$ or the $\mathrm{i}$-th readings of local times ${ }^{\mathrm{j}} \mathrm{t}$ and ${ }^{\mathrm{k}_{\mathrm{t}}}$ in nodes $\mathrm{j}$ and $\mathrm{k}$. To respect the implementation viewpoint, distributed applications consider for each node a distinctive local time, i.e. the time of a local physical clock that suits to measuring a duration of local process actions.

The primitive send(message,destination) implements asynchronous communication with non-blocking semantics. To respect different local clocks, the information transfer is controlled by a special clocking that is common for the source and the destination; however, the nodes communicate asynchronously by message passing through an input buffer at the destination. The input of a message induces the event for the related operation AWAIT(message,1). If any synchronization is required, it must be described explicitly using confirmation and the wait statements. In this case, additional attributes of time clarify the nature of the time component, ${ }^{{ }}$, of the node $j^{\prime}$ s behavior, ${ }^{j} \mathrm{P}=\left({ }^{j_{s}}\right.$, $\left.{ }^{j} t\right)$ : real-time distributed architecture enrich models of time by considering the number of time lines. A single time line suffices for global clocks while multiple time lines support independent local clocks. Accordingly, the values ${ }^{j_{i}}$ and ${ }^{k} t_{i}$ are either the $i$-th readings of global time, $t$, in nodes $j$ and $k$ or the $\mathrm{i}$-th readings of local times ${ }^{j_{t}}$ and ${ }^{k_{t}}$ in nodes $j$ and $k$. To respect the implementation viewpoint, distributed applications consider for each node a distinctive local time, i.e. the time of a local physical clock that suits to measuring a duration of local process actions.

The control structure primitives loop ... endloop delimit an indefinite cycle, which is exited upon a true result of testing the condition following the primitive when. Consequently, the statements, which occur between the primitives action and exit and which follow the endloop primitive, are executed. This combined statement enables to extend the language with additional control structures by simple macro-like text replacements, e.g.: In this case, additional attributes of time clarify the nature of the time component, ${ }^{j}$, of the node $j^{\prime}$ s behavior, ${ }^{j} \mathrm{P}=\left({ }^{j} s\right.$, $\left.{ }^{j} t\right)$ : real-time distributed architecture enrich models of time by considering the number of time lines. A single time line suffices for global clocks while multiple time lines support independent local clocks. Accordingly, the values ${ }^{{ }^{j}} t_{i}$ and ${ }^{k_{i}}$ are either the $\mathrm{i}$-th readings of global time, $\mathrm{t}$, in nodes $\mathrm{j}$ and $\mathrm{k}$ or the $i$-th readings of local times ${ }^{j} t$ and ${ }^{k} t$ in nodes $j$ and $k$. To respect the implementation viewpoint, distributed applications consider for each node a distinctive local time, i.e. the time of a local physical clock that suits to measuring a duration of local process actions.

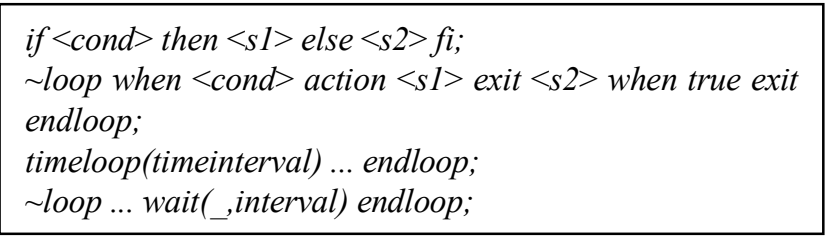

Actually, the control structure timeloop(timeinterval) ... endloop specifies an isochronous loop, which is periodically initiated whenever the timeinterval expires and which can be exited like the indefinite cycle. The operation AWAIT(local_ticks, timeinterval_value) defines the exact operational semantics of timing these initiations.

\section{CASE STUdies}

\section{A. Lift cabin Position Measurement}

The first case study consists in the logical structure description of the two-level structure, where higher level 
behaves as an event-driven component and lower level behaves as time-evolving interconnected component. The behaviour of the higher level component can be described by the following state sequence: In this case, additional attributes of time clarify the nature of the time component, ${ }^{j} t$, of the node $j^{\prime}$ s behavior, ${ }^{j} \mathrm{P}=\left({ }^{\mathrm{j}} \mathrm{s},{ }^{\mathrm{j}} \mathrm{t}\right)$ : real-time distributed architecture enrich models of time by considering the number of time lines. A single time line suffices for global clocks while multiple time lines support independent local clocks. Accordingly, the values ${ }^{j_{i}} t_{i}$ and ${ }^{k} t_{i}$ are either the $i$-th readings of global time, $t$, in nodes $j$ and $k$ or the $i$-th readings of local times ${ }^{j}$ and $k_{t}$ in nodes $j$ and $k$. To respect the implementation viewpoint, distributed applications consider for each node a distinctive local time, i.e. the time of a local physical clock that suits to measuring a duration of local process actions.

initialization $\rightarrow$ position_indication $\rightarrow$ fault_indication

The behaviour of the lower level can be described by three communicating, individually timed automata. The first automaton models the impulse detector, timed by its local clock that defines a sampling interval. This interval must conform not only to the maximal speed and the distance of position marks but also to a pattern of samples for impulse recognition, depending on the electro-magnetic interference characteristics of the environment. Let the fitting pattern, skipping possible transient fault states, is represented by '1100'. Then adequate behaviour for an impulse recognition can be described by the following sampling sequence with regular periodic timing: In this case, additional attributes of time clarify the nature of the time component, ${ }^{{ }} t$, of the node j's behavior, ${ }^{j} \mathrm{P}=\left({ }^{\mathrm{j}} \mathrm{s},{ }^{\mathrm{j}} \mathrm{t}\right)$ : real-time distributed architecture enrich models of time by considering the number of time lines. A single time line suffices for global clocks while multiple time lines support independent local clocks. Accordingly, the values ${ }^{j_{i}}$ and ${ }^{k} t_{i}$ are either the $\mathrm{i}$-th readings of global time, $t$, in nodes $\mathrm{j}$ and $\mathrm{k}$ or the $\mathrm{i}$-th readings of local times $j_{t}$ and $k_{t}$ in nodes $j$ and $k$. To respect the implementation viewpoint, distributed applications consider for each node a distinctive local time, i.e. the time of a local physical clock that suits to measuring a duration of local process actions.

$$
\mathrm{q}_{1} \stackrel{\text { inp }=1}{\rightarrow} \mathrm{q}_{2} \stackrel{\text { inp }=0}{\rightarrow} \ldots \mathrm{q}_{2} \stackrel{\text { inp }=1}{\rightarrow} \mathrm{q}_{3} \stackrel{\text { inp }=1}{\rightarrow} \ldots \mathrm{q}_{3} \stackrel{\text { inp }=0}{\rightarrow} \mathrm{q}_{4} \stackrel{\text { inp }=1}{\rightarrow} \mathrm{q}_{4} \stackrel{\text { inp }=0 / / M P}{\rightarrow} \mathrm{q}_{1}
$$

The information about detected impulse is sent to the counting automaton, which can also access the indication of the cabin movement direction through the variable $D$. The counting automaton communicates the position value to the display automaton. The display refreshment subsides to a timing mechanism dependent on the physiologic constants of human sight. In this case, additional attributes of time clarify the nature of the time component, ${ }^{{ }} \mathrm{t}$, of the node $\mathrm{j}$ 's behavior, ${ }^{\mathrm{j}} \mathrm{P}=\left(\mathrm{j}_{\mathrm{s}},{ }^{\mathrm{j}} \mathrm{t}\right)$ : real-time distributed architecture enrich models of time by considering the number of time lines. A single time line suffices for global clocks while multiple time lines support independent local clocks. Accordingly, the values ${ }^{j_{i}}$ and ${ }^{k} t_{i}$ are either the $i$-th readings of global time, $t$, in nodes $j$ and $k$ or the $i$-th readings of local times ${ }^{j}$ and ${ }^{k} t$ in nodes $j$ and $\mathrm{k}$. To respect the implementation viewpoint, distributed applications consider for each node a distinctive local time, i.e. the time of a local physical clock that suits to measuring a duration of local process actions.

For the sake of fault-detection requirements, the impulse generator and transfer path are doubled. Consequently, a second, identical impulse detector automaton appears necessary. The subsequent automaton is the reversible counter, which starts with the value $(\mathrm{h}+1) / 2$ and increments or decrements the value according to the "impulse detected" outputs from the first or second recognition automaton. Overflow or underflow of the preset values of $h$ or 1 indicate an error. In this case, additional attributes of time clarify the nature of the time component, ${ }^{j} t$, of the node $j$ 's behavior, ${ }^{\mathrm{j}} \mathrm{P}=\left({ }^{\mathrm{j}} \mathrm{s},{ }^{\mathrm{j}} \mathrm{t}\right)$ : real-time distributed architecture enrich models of time by considering the number of time lines. A single time line suffices for global clocks while multiple time lines support independent local clocks. Accordingly, the values ${ }^{j_{i}}$ and ${ }^{k} t_{i}$ are either the $i$-th readings of global time, $t$, in nodes $j$ and $k$ or the $i$-th readings of local times ${ }^{j}$ and ${ }^{k} t$ in nodes $j$ and $\mathrm{k}$. To respect the implementation viewpoint, distributed applications consider for each node a distinctive local time, i.e. the time of a local physical clock that suits to measuring a duration of local process actions. 


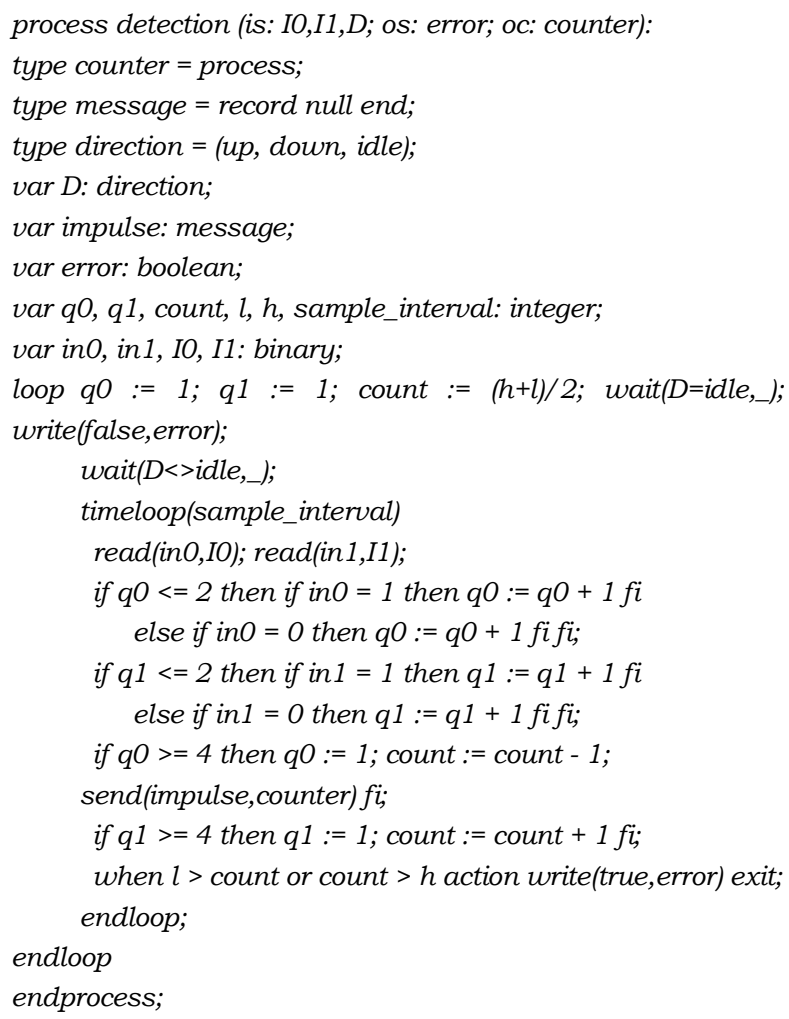

B. communication time measurement compatible with IEEE 1588

Cyber-physical systems can be highly connected and integrated in multiple ways, even across business operations and domain boundaries. Achieving effectively networked, cooperating, and human-interactive systems will be an integral factor in the adoption of such systems in the future.
Some of the key questions to be considered include what is needed to enable streamlined and predictable development, deployment, and evolution of networked and integrated cyber-physical systems, particularly as systems become interconnected with legacy systems and across industry boundaries.

The case study addresses important topics associated with the measurement of data communication delays in computer networks. The contribution consists in developing one-way delay measurement method and related support for Internet environment including monitoring and comparison of computer clocks, pulse-per-second signal processing, time server with guaranteed accuracy concepts and high accuracy time-stamping implementation.

Important characteristics of communication networks for respecting Real-time requirements deal namely with Transit Delay and Delay Variation with respect to actual Network Load. This case study considers packet switching networks, which in contrast to TDMA, FDMA or similar techniques, guarantee no limited transfer time. In fact, actual Transit Delay and Delay Variation depends on actual Network Load:

- Packet Transit Delay (PDT)- means packet delay due to data signal holdup, due to data processing by input or output in active devices, and during the period when packet is stored in buffers.

- Packet Delay Variation (PDV)- means the difference of individual packet's PDTs.

- Network Load (NL) - is directly influencing both PDT a PDV. Measuring NL and related values of PDT and PDV enables us to define functional dependencies for a particular network.

Evidently, this measurement, see Fig. 1, considers relative physical timing that facilitates clock synchronization using NTP and PTP protocols with precision better then 1 ms.

\section{CONCLUSIONS}

This paper stems from the author's research projects with partial results published in [16] ... [21]. The current paper addresses the role, interpretation and the deployment of the notion "time" in distributed cyber-physical systems. It discusses various possibilities how to approach such modeling and selects the fitting one, which enables to utilize the related specification language ASL in the domain applications specifications, modeling and measurements.

\section{ACKNOWLEDGMENT}

This project has been carried out with a financial support from the Czech Republic state budget by the IT4Innovations Centre of Excellence, EU, CZ 1.05/1.1.00/02.0070CEZ and by the MMT project no. MSM0021630528: SecurityOriented Research in Information Technology, by the Technological Agency of the Czech Republic through the grant no. TA01010632: SCADA system for control and monitoring RT processes, by the Ministry of Industry and Trade of the Czech Republic through the grant no. FRTI1/037: Automatic Attack Processing and by the Brno University of Technology, Faculty of Information 
Technology through the specific research grant no. FIT-S11-1: Advanced Secured, Reliable and Adaptive Information Technologies.

The author acknowledges contributions to the presented work by his colleagues Petr Matousek, Jaroslav Rab, Vladimir Vesely, Matej Gregr, Libor Polcak from the Faculty of Information Technology, and Radimir Vrba from the Faculty of Electrical Engineering and Communication.

\section{REFERENCES}

[1] D.W. Allan and N. Ashby and C. Hodge. Time in the Space Age. IEEE Spectrum vol.35, no.3, 2012, pp.42-51.

[2] R. Alur and T.A. Henzinger: Logics and Models of Real Time: A Survey. In: J.W. de Bakker et al. (eds.), Real-Time: Theory in Practice. Springer-Verlag, LNCS 600, 1992, pp.74-106.

[3] J.C.M. Baeten and J.A. Bergstra: Real space process algebra. In J.C.M. Baeten and J.F. Groote (eds), Concur 91, Springer-Verlag, LNCS 527, 1991, pp.96-110.

[4] J.C. Eidson, E.A. Lee, S.Matic, S.A. Seshia, J.Zou: A Time-Centric Model for Cyber-Physical Applications, MoDELS 2010, ACES-MB Workshop Proceedings, Oslo, Norway, October 4, 2006, pp.21-35.

[5] S.W. Hawking: A Brief History of Time--From Big Bang to Black Holes. Bantam Books, New York, 1988.

[6] A. Goswami and M. Joseph: Defining Time Domains for Computation. In: H.S.M. Zedan (ed.), Real-Time Systems, Theory and Applications. North-Holland, Amsterdam, 1990, pp.49-61.

[7] C.M. Holt: Intervals as Time Lattices. In: H. S. M. Zedan (ed.), RealTime Systems, Theory and Applications. North-Holland, Amsterdam, 1990, pp.63-79.

[8] R. Koymans. (Real) time: a philosophical perspective. In J.W. de Bakker, C. Huizing, W.P. de Roever, and G. Rozenberg (eds), RealTime: Theory in Practice, pp.353-370. Springer-Verlag, 1992. LNCS 600.

[9] B.H. Krogh, E. Lee, I. Lee, A. Mok, R. Rajkumar, L.R. Sha, A.S. Vincentelli, K. Shin, J. Stankovic, J. Sztipanovits, W. Wolf, W. Zhao: Cyber-Physical Systems, Executive Summary, CPS Steering Group, Washington D.C., March 6, 2008. [http://www.nsf.gov/pubs/2008/nsf08611/nsf08611.htm]

[10] M. Kudlek: Calendars and Chronologies. In C. Freksa, M. Jantzen, and R. Valk (eds), Foundations of Computer Science. SpringerVerlag, 1997. LNCS 1337.
[11] L. Lamport: Time, Clocks, and the Ordering of Events in a Distributed System, Communications of the ACM, vol.21, July 1978, pp.558-565.

[12] L. Lamport: Temporal Logic of Actions. ACM Transactions on Programming Languages and Systems, vol.16, no.3, 1994, pp.872923.

[13] E.A. Lee: Computing Needs Time, Communications of the ACM, vol.52, no.5, May 2009, pp.70-79.

[14] E.A. Lee: CPS Foundations, DAC'10, Anaheim, California, USA, ACM, June 2010, pp.737-742.

[15] S.E. Pomares Hernandez, J.R. Perez Cruz, M. Raynal, From the Happened-Before Relation to the Causal Ordered Set Abstraction, J. Parallel Distributed Computing, vol. 72, Feb. 2012, pp.791-795, doi:10.1016/j.jpdc.2012.02.015.

[16] O. Rysavy, M. Sveda, R. Vrba: A Framework for Cyber-Physical Systems Design - A Concept Study, Proceedings the Sixth International Conference on Systems ICONS 2012, Saint Gilles, Reunion Island, FR, IARIA, 2012, pp.79-82.

[17] M. Sveda: Local Time for Formal Specification of Networked Embedded System, In: WSEAS Transactions on Computers, Vol. 2, No. 1, 2003, Athens, GR, pp.4-9.

[18] M. Sveda: Rapid Prototyping of Networked Embedded Systems, In: Proceedings of the IEEE International Conference and Workshop on the Engineering of Computer-Based Systems 2003, Huntsville, AL, US, IEEE CS, 2003, pp.125-132,

[19] M. Sveda: A Design Framework for Internet-Based Embedded Distributed Systems, In: Proceedings of the International IEEE Conference and Workshop ECBS'2004, Los Alamitos, California, US, IEEE CS, 2004, pp.113-120

[20] M. Sveda, R. Vrba: An Embedded Application Regarded as CyberPhysical System, Proceedings of the Fifth International Conference on Systems ICONS 2010, Les Menuires, FR, IEEE CS, 2010, pp.170174 .

[21] M. Sveda, R. Vrba: A Cyber-Physical System Design Approach, Proceedings of the Sixth International Conference on Systems ICONS 2011, St. Maarten, AN, IARIA, 2011, pp.12-18.

[22] N. Wiener. Cybernetics or Control and Communication in the Animal and the Machine. Wiley, New York, 1948.

[23] S. Ying et al.: Foundations for Innovation in Cyber-Physical Systems, Workshop Report, Energetics Incorporated, Columbia, Maryland, US, January 2013.

[24] J. Zwiers: Layering and Action Refinement for Timed systems. In J.W. de Bakker, C. Huizing, W.P. de Roever, and G. Rozenberg (eds.), Real-Time: Theory in Practice Springer-Verlag, LNCS 600, 1992, pp.687-723.

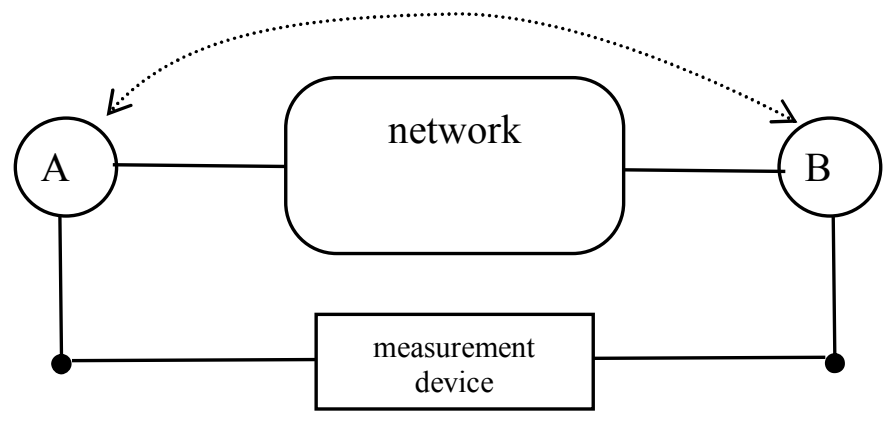

Figure 1. Example of a "one way delay" configuration for communication time measurement compatible with IEEE 1588.

\section{Creative Commons Attribution License 4.0 (Attribution 4.0 International, CC BY 4.0)}

This article is published under the terms of the Creative Commons Attribution License 4.0 https://creativecommons.org/licenses/by/4.0/deed.en_US 